\title{
Relationship between body mass index and hippocampal glutamate/glutamine in bipolar disorder*
}

David J. Bond, Leonardo Evangelista da Silveira, Erin L. MacMillan, Ivan J. Torres, Donna J. Lang, Wayne Su, William G. Honer, Raymond W. Lam and Lakshmi N. Yatham

\section{Background}

We previously reported that patients with early-stage bipolar disorder, but not healthy comparison controls, had body mass index (BMI)-related volume reductions in limbic brain areas, suggesting that the structural brain changes characteristic of bipolar disorder were more pronounced with increased weight.

\section{Aims \\ To determine whether the most consistently reported neurochemical abnormality in bipolar disorder, increased glutamate/glutamine (GIX), was also more prominent with higher BMI.}

\section{Method}

We used single-voxel proton magnetic resonance spectroscopy to measure hippocampal GIX in 51 patients with first-episode mania (mean $\mathrm{BMI}=24.1$ ) and 28 healthy controls (mean $\mathrm{BMI}=23.3$ )

\section{Results}

In patients, but not healthy controls, linear regression demonstrated that higher BMI predicted greater GIX. Factoria ANCOVA showed a significant $\mathrm{BMI} \times$ diagnosis interaction, confirming a distinct effect of weight on GlX in patients.

\section{Conclusions}

Together with our volumetric studies, these results suggest that higher BMI is associated with more pronounced structural and neurochemical limbic brain changes in bipolar disorder, even in early-stage patients with low obesity rates.

\section{Declaration of interest}

D.J.B. has received speaking fees or sat on advisory boards for: the Canadian Network for Mood and Anxiety Treatments (CANMAT), the Canadian Psychiatric Association, Pfizer, Sunovion, Bristol-Myers Squibb, Otsuka, AstraZeneca, Janssen-Ortho and Myriad; and has received research support from: the Canadian Institutes of Health Research (CIHR), the UBC Institute of Mental Health/Coast Capital Depression Research Fund and Pfizer. W.G.H. has received consulting fees or sat on paid advisory boards for: $\mathrm{MDH}$ Consulting, In Silico, Novartis, Lilly, Lundbeck and Roche; received honouraria from Rush University, the Korean Society for Schizophrenia Research, the Centre for Addiction and Mental Health (Toronto, Ontario), the British Columbia Schizophrenia Society, the Fraser, Providence and Vancouver Coastal Health Authorities, and the Canadian Agency for Drugs and Technology in Health; and received grants from CIHR. R.W.L. is on speaker/advisory boards for, or has received research grants from: AstraZeneca, Bristol-Myers Squibb, CIHR, Canadian Psychiatric Association, Canadian Psychiatric Association Foundation, CANMAT, Eli Lilly, Litebook Company, Lundbeck, Lundbeck Institute, Merck, Mochida, Pfizer, Servier, St Jude Medical, UBC Institute of Mental Health/Coast Capital Savings, Takeda and Wyeth. L.N.Y. is on speaker/advisory boards for, or has received research grants from: AstraZeneca, Bristol-Myers Squibb, CIHR, CANMAT, Eli Lilly, GlaxoSmithKline, Janssen, the Michael Smith Foundation for Health Research, Pfizer, Servier and the Stanley Foundation

\section{Copyright and usage}

(c) The Royal college of Psychiatrists 2016.
Over two billion adults worldwide are overweight or obese. ${ }^{1}$ As prevalent as obesity is in the general population, it disproportionately affects people with bipolar disorder. In North American studies, obesity rates in people with bipolar disorder ranged from $22 \%$ to $53 \%$ compared with $9-32 \%$ in people without bipolar disorder. ${ }^{2-4}$ European and Asian patients had lower rates of $10-41 \%$, but these were still elevated compared with the $5-32 \%$ reported in non-psychiatric comparator groups from the same countries. ${ }^{5-7}$ The largest study to date reported that people with bipolar disorder were two-thirds again more likely to be obese than the age-, gender- and ethnically adjusted general population. ${ }^{8}$ Adipose tissue is a key endocrine organ that plays a critical role in regulating metabolism and immune functioning. It does so by producing a plethora of systemically acting molecules - over 50 have been identified - including adipokines such as leptin and

*A modified version of this report was presented at the 9th International Conference on Bipolar Disorders, 9-11 June 2011, Pittsburgh, Pennsylvania, USA. adiponectin, and cytokines such as tumour necrosis factor-alpha (TNF- $\alpha)$ and interleukins. As weight increases and adipose tissue expands, it becomes infiltrated with activated macrophages, leading to increased production of inflammatory, oxidative and thrombotic factors such as TNF- $\alpha$, leptin, peroxynitrite and plasminogen activator inhibitor-1, and reduced synthesis of anti-inflammatory molecules such as interleukin-10 and adiponectin. ${ }^{9}$ This proinflammatory, oxidative, hypercoagulable state has been causally linked to the development of obesityrelated medical conditions such as diabetes. ${ }^{10}$ Converging evidence suggests that the brain is also susceptible to obesity-related damage. People who are obese have reduced grey matter volume compared with normal weight individuals, a finding that was recently demonstrated in children as young as $5 .{ }^{11}$ Obesity-related cognitive impairments are also detectable throughout the lifespan, ${ }^{12}$ and people who are overweight or obese in mid-life have 35\% and $104 \%$ increased risks, respectively, of developing Alzheimer's disease. ${ }^{13}$ Finally, animals assigned to experimental weight gain conditions develop pathological brain changes, ${ }^{14}$ whereas weight 
loss interventions in humans lead to greater improvement in cognition than control conditions, ${ }^{15}$ suggesting a causal relationship between weight and brain structure and function.

In keeping with the adverse brain effects of obesity, patients with bipolar disorder who are obese experience a more severe mood illness than those patients who are normal weight, including more frequent mood episodes, lower response rates to moodstabilising medications, more suicide attempts and greater interepisode cognitive impairment. ${ }^{16-18}$ However, the mechanisms by which obesity has an impact on clinical outcomes in bipolar disorder are unclear. This led us, and others, to investigate whether elevated body mass index (BMI) is associated with abnormalities in brain regions relevant to bipolar disorder. Several magnetic resonance imaging (MRI) studies have since reported that patients with first-episode mania, but not healthy comparator controls, had BMI-related grey and white matter volume reductions and reduced white matter integrity in limbic brain areas implicated in bipolar disorder. ${ }^{19,20}$ Thus, elevated BMI is associated with unique brain changes early in bipolar disorder, such that the structural brain changes characteristic of the illness are more prominent in overweight/obese patients. The current study extends these findings by examining whether the most consistently reported neurochemical abnormality in bipolar disorder, increased glutamate/glutamine $(\mathrm{Glx})$, was also more pronounced with elevated BMI. We used single-voxel proton magnetic resonance spectroscopy $\left({ }^{1} \mathrm{H}\right.$-MRS) to investigate the relationship between BMI and hippocampal Glx in patients with bipolar disorder at recovery from their first manic episode, and a comparison group of healthy controls. We focused on the hippocampus as it is implicated in the pathophysiology of bipolar disorder and our MRI studies suggested that temporal lobe structures may be particularly susceptible to BMI-related changes. We chose to investigate Glx because it is the most extensively studied neurochemical signature in bipolar disorder, and a meta-analysis of ${ }^{1} \mathrm{H}$-MRS studies demonstrated that Glx activity is elevated in patients. ${ }^{21}$ Moreover, elevated glutamate levels are neurotoxic, and BMI-related Glx increases thus suggest a possible explanation for the reduced brain volumes we previously observed. We therefore hypothesised that higher BMI would be associated with greater hippocampal Glx in patients, but not healthy controls.

\section{Method}

\section{The Systematic Treatment Optimization Program for Early Mania (STOP-EM)}

STOP-EM is a comprehensive study of clinical outcomes, brain morphology and neurochemistry in patients with bipolar disorder with a first episode of mania. A detailed description of the programme was published previously. ${ }^{22}$ Briefly, patients with bipolar disorder aged 14-35 who experienced their first DSM-IV-TR-defined manic episode ${ }^{23}$ within the previous 3 months were recruited from the University of British Columbia (UBC) Hospital Mood Disorders Clinical Research Unit and affiliated sites (the bipolar group). Patients may have had a pure or mixed mania, with or without psychosis, and with or without comorbid conditions. They received treatment for bipolar disorder according to Canadian clinical practice guidelines. ${ }^{24}$ Healthy comparison controls aged 14-35 were recruited from the greater Vancouver metropolitan area through print advertisements and online forums such as Craigslist (control group). The UBC Clinical Research Ethics Board approved the procedures described here, and written informed consent was obtained prior to any study activities taking place.

\section{Clinical assessments}

At enrolment, the diagnoses of bipolar disorder and first manic episode were based on a comprehensive interview with an academic research psychiatrist and confirmed with the Mini International Neuropsychiatric Interview (MINI) ${ }^{25}$ The control group were administered the MINI and were enrolled if they had no personal or self-reported family history of psychiatric illness in first- or second-degree relatives. Sociodemographic and clinical data were collected using a standardised protocol. Mood and psychotic symptoms were quantified with the Young Mania Rating Scale (YMRS), ${ }^{26}$ the Montgomery-Åsberg Depression Rating Scale (MADRS) ${ }^{27}$ and the Brief Psychiatric Rating Scale (BPRS). ${ }^{28}$ Participants were weighed in a non-fasting state in light clothing with footwear removed. BMI was calculated as weight $(\mathrm{kg}) /$ height $\left(\right.$ metres $\left.^{2}\right)$. Underweight was defined as $\mathrm{BMI}<18.50$, normal weight as BMI 18.50-24.99, overweight as BMI 25.00-29.99 and obese as BMI $\geqslant 30.00$.

\section{MRI/MRS protocols and data extraction}

$T_{1}$-weighted magnetic resonance images were acquired with a Philips Achieva 3.0 Tesla scanner (Amsterdam, The Netherlands), typically on the same day as clinical assessment, using a three-dimensional axial inversion recovery-weighted spoiled gradient recalled sequence with the following parameters: field of view $(\mathrm{FOV})=25.6 \mathrm{~cm}$, matrix: $256 \times 256$, isotropic voxels $\left(1 \times 1 \times 1 \mathrm{~mm}^{3}\right)$, autoshim, repetition time (TR)/echo time $(\mathrm{TE})=$ autoset shortest, transmit/receive head coil, flip angle: $8^{\circ}$, sensitivity encoding (SENSE) $=0$, and $1 \mathrm{~mm}$ thick contiguous 180 slices of the whole brain. Hippocampal volumes were estimated using Freesurfer v5.1 subcortical segmentation. The grey matter, white matter and cerebrospinal fluid (CSF) composition of the MRS voxel was determined with the FSL v4.1.9 FAST tool (FMRIB Software Library, www.fmrib.ox.ac.uk/fsl/). ${ }^{29,30}$

MRS signals were acquired with the Philips $3.0 \mathrm{~T}$ unit. $T_{2}$-weighted coronal, sagittal and axial images for anatomical parameters were first obtained. A point resolved spectroscopy (PRESS) sequence $(\mathrm{TE}=35 \mathrm{~ms}, \mathrm{TR}=2000 \mathrm{~ms}$ ) was then used to acquire data from $30 \mathrm{~mm} \times 15 \mathrm{~mm} \times 15 \mathrm{~mm}$ voxels in the hippocampus on both sides of the brain. Using the sagittal image, the voxel was placed with the long axis angled along the hippocampus. Its position in the medial/lateral and superior/inferior directions was adjusted based on the coronal and axial images to include the maximum amount of hippocampus and avoid CSF. Voxel placement and tissue composition are shown in online Fig. DS1 $(a-c)$. Chemical shift selective (CHESS) pulses were used to suppress the water signal during data acquisition, and water-unsuppressed signals were also obtained for eddy current correction and to reference metabolite signals. In total, 128 water-suppressed and 16 nonwater-suppressed averages were acquired from each voxel.

The concentrations of Glx were extracted using LCModel v. 6.3 and normalised to the unsuppressed water spectrum. Sample ${ }^{1} \mathrm{H}$-MRS spectra from a patient and a healthy control, showing typical data quality, are displayed in online Fig. DS1(d-e). Glx-to-water ratios were converted to institutional absolute concentrations in millimolar units by correcting for the mean water concentration of the voxel, taking into account the fractions of grey matter, white matter and CSF in the voxel and their respective water concentrations, and water and Glx signal $T_{1}$ and $T_{2}$ relaxation during the acquisition (see online supplement DS1). Values for water and Glx $T_{1}$ and $T_{2}$ relaxation times were taken from the literature. ${ }^{31}$

\section{Data analysis and statistics}

Statistical analyses were carried out using IBM SPSS Statistics for Windows 19.0. Comparisons were two-tailed, with a significance 
level of $\alpha=0.05$. We examined sociodemographic and clinical variables with $t$-tests, factorial ANOVA, $\chi^{2}$-tests or log-linear analysis as appropriate. For our primary analysis, we constructed linear regression models separately for the bipolar and control groups, with mean $(\operatorname{left}(\mathrm{L})+\operatorname{right}(\mathrm{R}) / 2)$ hippocampal Glx as the dependent variable. Predictors were entered using the forced-entry method. For patients, they included BMI, age, YMRS and MADRS scores, and treatment with lithium, valproate semisodium and second-generation antipsychotics (in chlorpromazine equivalents). To ensure that BMI-related differences in the composition of the MRS voxel did not have an impact on our results, mean $(\mathrm{L}+\mathrm{R} / 2)$ hippocampal tissue volume in the voxel was also entered as a predictor. For the control group, predictors included BMI, age, and mean voxel hippocampal volume. The primary analysis allowed us to determine whether the continuous variable of BMI significantly predicted mean Glx, and importantly, allowed us to confirm this in patients when potentially confounding clinical and treatment variables were controlled for.

As a secondary analysis, we used factorial ANCOVA to assess the impact of diagnosis (bipolar $v$. control group), BMI category (normal weight $v$. overweight/obese) and their interaction on mean Glx. We covaried this analysis for age and mean voxel hippocampal volume. The secondary analysis enabled us to investigate whether the impact of weight on hippocampal neurochemistry differed based on diagnosis. Participants with Cramer-Rao lower bounds $>25 \%$ for Glx were excluded from the primary and secondary analyses.

\section{Results}

\section{Bipolar and control groups}

In total, 57 of 71 patients and 31 of 42 healthy controls enrolled in STOP-EM had baseline BMI and neurochemical data. Participants with missing data had either declined to be weighed or to undergo the MRS procedure, or had their MRS data discarded because of artefacts. Of those with complete data, six patients and three controls were excluded for having Cramer-Rao lower bounds $>25 \%$ for Glx, leaving 51 patients and 28 healthy controls for our analyses. Reflecting their early illness stage and short duration of pharmacotherapy, the bipolar group did not differ from the control group with respect to mean BMI $(24.11$ (s.d.=4.12) $v$. 23.27 (s.d. $=2.92$ ); $F=0.910$, d.f. $=1, P=0.343$ ), the proportions with normal weight $(66.7 \%$ v. $78.6 \%)$, overweight $(23.5 \% v$. $17.9 \%)$ or obesity $(9.8 \%$ v. $3.6 \%)\left(\chi^{2}=1.556\right.$, d.f. $\left.=2, P=0.459\right)$ or mean hippocampal Glx $(2.3 \%$ greater in the bipolar group;
13.52 (s.d.=2.38) v. $13.21 \quad$ (s.d.=2.95) $\mathrm{mmol} / \mathrm{L}, \quad F=0.106$, d.f. $=1, P=0.742)$.

\section{BMI and hippocampal GIX}

Overweight/obese and normal weight patients and controls were well-matched on sociodemographic and clinical characteristics (Tables 1 and 2), and had similar mean hippocampal volumes and amounts of hippocampal and non-hippocampal grey and white matter in the MRS voxel (online Table DS1). Measures of MRS data quality, including signal-to-noise ratio (SNR) and full-width at half maximum (FWHM), also did not differ between the groups (Table DS1).

\section{Linear regression models}

In the bipolar group, higher BMI significantly predicted greater Glx $(\alpha=0.309, t=2.036, P=0.048)$ (Table 3 and Fig. 1 ). No other sociodemographic, clinical or treatment variables were associated with Glx. When we repeated our analysis excluding an outlying patient with a BMI of 42.19 , BMI remained a significant predictor of Glx ( $\beta=0.382, t=2.603, P=0.013)$. In the control group, there was a non-significant negative relationship between BMI and Glx $(\beta=-0.046, t=-0.223, P=0.826)$ and no other significant predictors.

\section{Factorial ANCOVA}

Factorial ANCOVA did not detect a main effect of either diagnosis or BMI on hippocampal neurochemistry (diagnosis: $F=1.057$, d.f. $=1, P=0.307$; BMI: $F=1.904$, d.f. $=1, P=0.172$ ). However, there was a significant $\mathrm{BMI} \times$ diagnosis interaction $(F=4.142$, d.f. $=1, P=0.045$ ), indicating the effect of weight on Glx differed in the patient and control groups. This remained significant when the outlying patient was excluded $(F=4.033$, d.f. $=1, P=0.048)$. Follow-up ANCOVAs demonstrated that in the bipolar group Glx was $17.6 \%$ greater in those who were overweight/obese than in those who were normal weight $(15.02$ (s.d.=2.30) v. 12.77 (s.d.=2.06) $\mathrm{mmol} / \mathrm{L}, F=13.178$, d.f. $=1, P=0.001$ ), with a large effect size (Cohen's $d$ ) of 1.03 (Fig. 2). In contrast, in the control group Glx was 3.6\% less in those who were overweight/obese than in those who were normal weight $(12.82$ (s.d.=1.32) v. 13.31 (s.d. $=3.28) \mathrm{mmol} / \mathrm{L}, F=0.091$, d.f. $=1, P=0.765)$, with a small effect size of -0.20 (Fig. 2).

Table 1 Sociodemographic characteristics of the bipolar disorder and control groups ${ }^{a}$

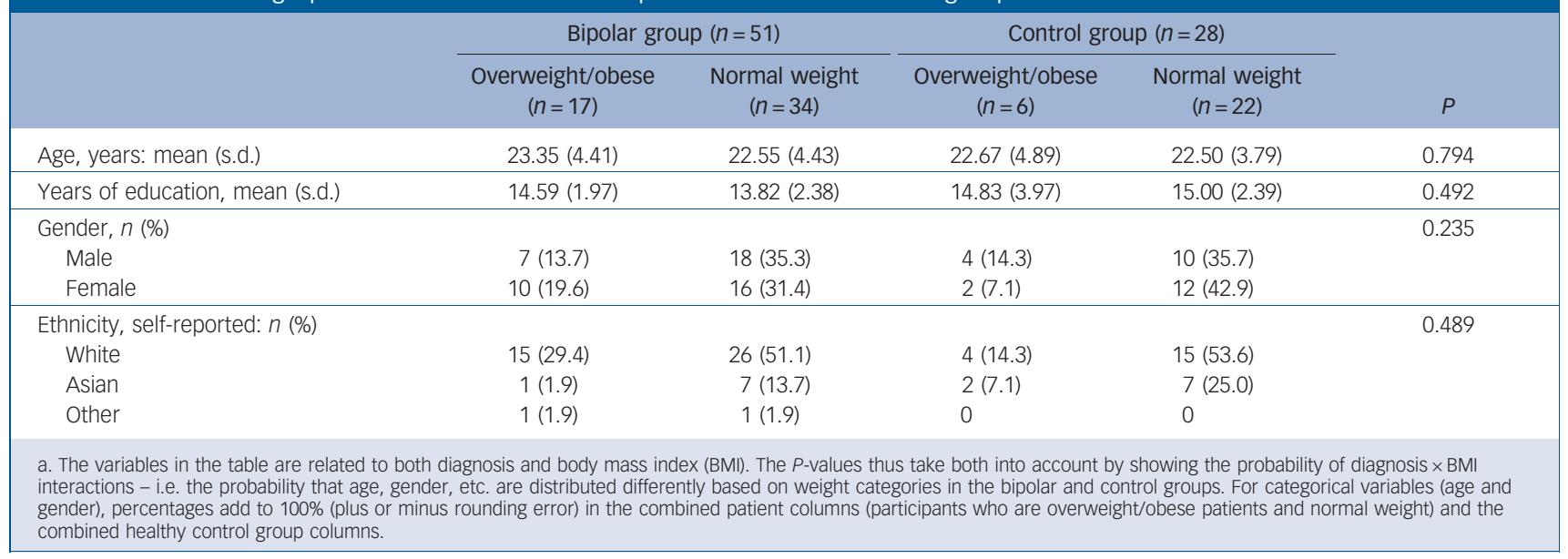




\begin{tabular}{|c|c|c|c|}
\hline & Overweight/obese $(n=17)$ & Normal weight $(n=34)$ & $P$ \\
\hline \multicolumn{4}{|l|}{ Baseline rating scale scores, mean (s.d.) } \\
\hline YMRS & $2.71(3.08)$ & $3.53(6.21)$ & 0.619 \\
\hline MADRS & $5.88(7.66)$ & $6.12(8.61)$ & 0.919 \\
\hline $\mathrm{BPRS}^{\mathrm{a}}$ & $21.88(3.98)$ & $22.67(6.32)$ & 0.647 \\
\hline Duration of manic/mixed episode, days: mean (s.d.) & $56.23(43.95)$ & $64.26(55.00)$ & 0.606 \\
\hline Duration of mood disorder, years: mean (s.d.) & 2.24 (3.09) & $3.03(4.84)$ & 0.543 \\
\hline In remission (YMRS < 20 and MADRS $\leqslant 20), n(\%)$ & $16(94.1)$ & $29(85.3)$ & 0.654 \\
\hline Previous depressive episode, $n$ (\%) & $8(47.1)$ & $17(50.0)$ & 0.838 \\
\hline Previous hypomanic episode, $n$ (\%) & $5(29.4)$ & $5(14.7)$ & 0.193 \\
\hline \multicolumn{4}{|l|}{ Comorbidity, $n(\%)$} \\
\hline Anxiety ${ }^{a}$ & 0 & $4(12.5)$ & 0.169 \\
\hline Alcohol misuse or dependence ${ }^{b}$ & $5(29.4)$ & $4(12.1)$ & 0.128 \\
\hline Cannabis misuse or dependence ${ }^{b}$ & $7(41.2)$ & $10(30.3)$ & 0.437 \\
\hline Other drug misuse or dependence ${ }^{b}$ & 0 & $4(12.1)$ & 0.183 \\
\hline Psychosis during initial mania, $n(\%)$ & $12(70.6)$ & $25(73.5)$ & 0.536 \\
\hline \multicolumn{4}{|l|}{ Medication, $n(\%)$} \\
\hline Lithium & $5(29.4)$ & $16(47.1)$ & 0.229 \\
\hline Valproate semisodium & $12(70.6)$ & $14(41.2)$ & 0.046 \\
\hline Risperidone & $7(41.2)$ & $15(44.1)$ & 0.844 \\
\hline Olanzapine & $3(17.6)$ & $6(17.6)$ & 0.655 \\
\hline Quetiapine & $4(23.5)$ & $6(17.6)$ & 0.444 \\
\hline Mood stabiliser+antipsychotic & $14(82.4)$ & $25(73.5)$ & 0.368 \\
\hline No medication & 0 & $3(8.8)$ & 0.285 \\
\hline
\end{tabular}

\section{Discussion}

\section{Main findings}

Our results show a relationship between increased weight and altered neurochemistry early in the course of bipolar disorder. Higher BMI was associated with greater hippocampal Glx in patients at recovery from their first manic episode - the first time, in other words, that they could be diagnosed with bipolar disorder. Our findings are particularly noteworthy considering that patients' mean BMI was well within the normal range; that only a third were overweight/obese; and that three-quarters of overweight/obese participants were in fact overweight. This suggests that weight-related neurochemical changes affect patients with bipolar disorder across a broad range of BMIs, not just BMIs in the obese range. Together with our previous volumetric MRI studies, these results suggest that the structural and chemical brain changes typical of bipolar disorder are more pronounced with higher BMI.

The association between BMI and Glx was surprisingly robust - the difference in Glx between those in the bipolar group who were overweight/obese compared with normal weight $(17.6 \%$ higher) was over seven times greater than the difference between the entire bipolar group and the entire control group $(2.3 \%$ higher). In contrast, there was not a significant relationship between BMI and Glx in the control group. Whether this was expected is unknown, since no previous studies have examined the relationship between BMI and hippocampal Glx in healthy individuals, or between BMI and Glx in any brain region in healthy individuals in the same age range as ours. Although our sample included a smaller number of healthy controls than patients, resulting in reduced statistical power to detect such a relationship, we are confident that the findings in our patients

Table 3 Regression analyses showing the relationship between body mass index (BMI) and mean bilateral hippocampal glutamate/ glutamine (GIX) in the bipolar disorder and control groups ${ }^{\mathrm{a}}$

\begin{tabular}{|c|c|c|c|}
\hline & \multicolumn{3}{|c|}{ Glutamate/glutamine (Glx) } \\
\hline & $\beta$ & $t$ & $P$ \\
\hline \multicolumn{4}{|l|}{ Bipolar group } \\
\hline Body mass index & 0.309 & 2.036 & 0.048 \\
\hline Age & -0.208 & -1.450 & 0.154 \\
\hline Young Mania Rating Scale score & 0.010 & 0.070 & 0.945 \\
\hline Montgomery-Åsberg Depression Rating Scale score & 0.027 & 0.176 & 0.862 \\
\hline Lithium use & 0.226 & 0.961 & 0.342 \\
\hline Valproate semisodium use & -0.084 & -0.347 & 0.731 \\
\hline Second-generation antipsychotic use (chlorpromazine equivalents) & 0.091 & 0.608 & 0.547 \\
\hline Hippocampal volume in voxel & -0.070 & -0.473 & 0.639 \\
\hline \multicolumn{4}{|l|}{ Control group } \\
\hline Body mass index & -0.046 & -0.223 & 0.826 \\
\hline Age & -0.028 & -0.138 & 0.891 \\
\hline Hippocampal volume in voxel & -0.196 & -0.971 & 0.341 \\
\hline
\end{tabular}




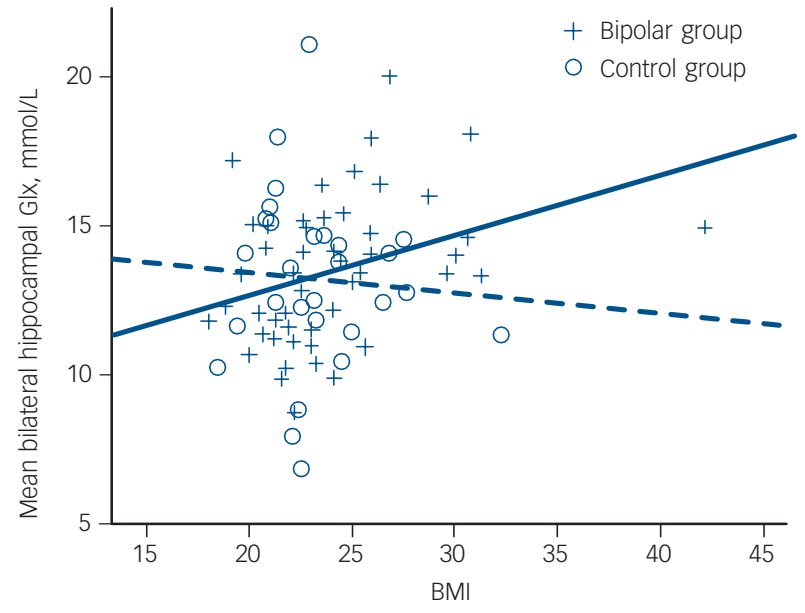

Fig. 1 Relationship between body mass index (BMI) and mean bilateral hippocampal glutamate/glutamine (GIX) in the bipolar and control groups.

Bipolar group: $\beta=0.309, P=0.048$; control group: $\beta=-0.046, P=0.826$.

are unique to bipolar disorder since our factorial ANCOVA detected a BMI $\times$ diagnosis interaction for Glx. Moreover, although non-significant, the direction of the relationship was reversed in the control group - i.e. increased BMI was associated with lower Glx.

\section{Importance of GIX}

Glutamate is the primary excitatory neurotransmitter in the central nervous system (CNS), and also plays important roles in synaptic plasticity and memory formation. After being released into the synapse, it is taken up by astrocytes and converted to glutamine, which is shuttled back to the presynaptic neuron, where glutamate is resynthesised from it. When glutamate is present in excessive amounts, it activates inotropic receptors in extra-synaptic sites and causes neurotoxicity via calcium influx, nitric oxide synthesis and free radical generation. Meta-analytic data show that increased Glx is the most consistently reported neurochemical alteration in bipolar disorder. ${ }^{21}$

\section{Findings from other studies}

Our finding of a BMI-related increase in Glx in a bipolar group with a low rate of obesity is in keeping with a large body of evidence demonstrating that the adverse health consequences of elevated BMI begin in the overweight range. One pooled analysis of 89 studies found a 'dose-response' relationship between BMI and hypertension, diabetes, ischaemic heart disease, stroke and various cancers, such that the prevalence of these conditions was increased in overweight people (hazard ratios (HRs) $=1.15-3.92$ ) and further increased in obese people (HRs $=1.49-12.42) .{ }^{32}$ Two large meta-analyses, including a combined total of 2.4 million participants, reported similar dose-response relationships between BMI and all-cause mortality over $8-10$ years. ${ }^{33,34}$ The HRs for early mortality were 1.03 for mild overweight (BMI 25-27.4), 1.11 for moderate overweight (BMI 27.9-29.9), 1.25 for class I obesity (BMI 30-34.9), 1.59 for class II obesity (BMI 35-39.9), and 1.99 for class III obesity (BMI 40-49.9). ${ }^{34}$ A recent study of $n=12664$ adolescents and young adults with a similar age range and BMI distribution to our sample found that a higher BMI within the non-obese range $(\mathrm{BMI}<30)$ was associated with significant increases in multiple health risk biomarkers, including lipoproteins, monounsaturated and

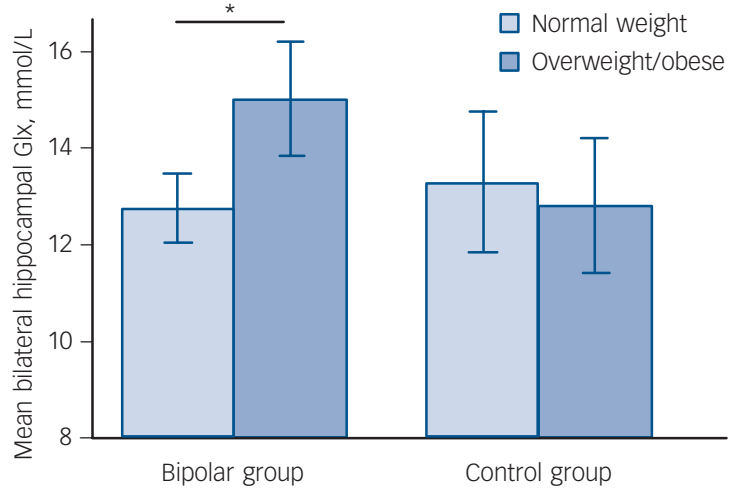

Fig. 2 Mean (95\% confidence interval) bilateral hippocampal glutamate/glutamine (Glx) in participants who are overweight/ obese and normal weight in the bipolar disorder and control groups.

Vertical bars indicate $95 \%$ confidence intervals. ${ }^{*} P=0.001$.

saturated fatty acids, branched-chain aromatic amino acids, inflammatory markers and adipokines. ${ }^{35}$ These results largely held true even when the analysis was confined to normal weight individuals $(\mathrm{BMI}<25)$.

\section{Possible explanations for our findings}

Our MRI and MRS findings suggesting greater limbic brain changes in those in the bipolar group who were overweight/obese have potentially profound relevance for understanding the neurobiology of the illness. Traditional theories of mood dysregulation emphasise structural and functional changes in emotion-generating and -regulating brain areas, and aberrations in the monoamine neurotransmitters that modulate their activity. More recent theories also propose central roles for inflammation, mitochondrial dysfunction and alterations in adipokine levels. Bipolar disorder, like obesity, is an inflammatory condition, with the ratio of serum inflammatory to anti-inflammatory cytokines increased during manic and depressive episodes, but relatively normalised during euthymia. ${ }^{36}$ Serum inflammation has an impact on the brain via transport mechanisms for cytokines across the blood-brain barrier and the presence of cytokine receptors on the vagus nerve and the epithelial cells of the blood-brain barrier. ${ }^{37}$ Moreover, inflammation in limbic brain areas in patients with bipolar disorder has been demonstrated in post-mortem brain samples and in vivo in a PET imaging study. ${ }^{38,39}$ Persisting obesity-related inflammation during euthymia is thus one possible mechanism to explain the more prominent neurobiological changes in patients with bipolar disorder who are obese.

Aberrations in appetite control hormones may also be relevant to the pathophysiology of bipolar disorder. Alterations in serum adipokines, such as leptin and ghrelin, are a well-known complication of obesity, ${ }^{9}$ and have also been reported in patients with bipolar disorder. ${ }^{40}$ Receptors for these molecules are widely distributed throughout limbic brain areas such as the frontal and temporal lobes and the midbrain. ${ }^{41,42}$ raising the possibility that they broadly subserve brain reward circuits relevant to bipolar disorder. Furthermore, adipokines have neuroprotective effects, ${ }^{43}$ promote hippocampal plasticity ${ }^{44}$ and modulate dopamine activity ${ }^{45}$ all functions germane to bipolar disorder. Leptin has direct effects on mitochondrial activity, ${ }^{46}$ which is particularly relevant to our current results since glutamate is produced in the mitochondrial matrix. Obesity-related biochemical alterations may thus lie at the nexus of inflammation, mitochondrial 
dysfunction and brain reward circuits, which is increasingly believed to be important to the pathophysiology of bipolar disorder.

\section{Limitations}

Our results must be interpreted in light of the limitations of our study. The most important is its naturalistic, cross-sectional design, which precludes us from determining whether there is a causal relationship between BMI and neurochemistry. Other possible explanations for our findings include a subtype of bipolar disorder characterised by both a propensity for weight gain and more pronounced neurochemical changes; and a confounding effect of medications on both weight and neurochemistry, creating an artefactual association between them. However, we note that the association between BMI and Glx was detected in our primary analysis when medication use was controlled for, and that no medications were significantly associated with Glx. Nonetheless, the question of whether elevated BMI causes adverse clinical and neurobiological outcomes in bipolar disorder is among the most pressing to arise from this line of research.

Additional limitations include that rates of overweight and obesity were low in our participants. However, our regression analyses demonstrated that increases in the continuous variable of BMI were associated with similar outcomes as dichotomised overweight/obesity, suggesting that our results in fact hold across the range of BMIs in our sample. We did not gather data on central obesity, or components of the metabolic syndrome, and we were therefore unable to examine whether these measures of adiposity were independently associated with Glx. We also did not systematically collect data on factors that might moderate the impact of obesity on neurochemistry, such as diet and exercise. Finally, our results present the measurement of the combined signal from glutamate and glutamine, preventing the interpretation of either compound individually. Nonetheless, their combined measurement can be interpreted as indicative of the overall activity of the glutamate-glutamine cycle.

\section{Directions for further study}

This is the first report to demonstrate that higher BMI is associated with greater Glx in bipolar disorder, or any psychiatric illness. Further studies are needed to confirm our findings, and to determine whether they hold true in untreated patients, those with longer illness durations and those in acute mood episodes. It would also be of interest to investigate whether the relationship between BMI and Glx is present in individuals at risk for bipolar disorder, such as those with family members with the illness. Finally, additional investigations should be carried out to characterise the impact of weight on neurochemistry in other psychiatric illnesses with high obesity rates and alterations in Glx, such as major depressive disorder and schizophrenia.

\footnotetext{
David J. Bond, MD, PhD, Mood Disorders Centre, University of British Columbia, Vancouver, Canada and Department of Psychiatry, University of Minnesota, Minneapolis, USA; Leonardo Evangelista da Silveira, MD, Mood Disorders Centre University of British Columbia, Vancouver, Canada, Laboratory of Molecular Psychiatry, Centro de Pesquisas Experimentais, Hospital de Clínicas de Porto Alegre, Porto Alegre and INCT for Translational Medicine, Porto Alegre, Brazil: Erin L. MacMillan, PhD, Department of Medicine (Neurology), University of British Columbia, Vancouver, Canada; Ivan J. Torres, PhD, Mood Disorders Centre, Columbia, Vancouver, Canada; Ivan J. Torres, PhD, Mood Disorders Centre,
University of British Columbia, Vancouver, Canada; Donna J. Lang, PhD, Wayne Su, University of British Columbia, Vancouver, Canada; Donna J. Lang, PhD, Wayne
MSc, William G. Honer, MD, Centre for Complex Disorders, University of British Columbia, Vancouver, Canada; Raymond W. Lam, MD, Lakshmi N. Yatham, MBBS, Mood Disorders Centre, University of British Columbia, Vancouver, Canada

Correspondence: Lakshmi N. Yatham, MBBS, MBA (Exec), FRCPC, MRCPsych (UK), Department of Psychiatry, University of British Columbia, Room 2C7-2255 Wesbrook Mall, vancouver, BC, Canada V6T 2A1. Email: yatham@mail.ubc.ca

First received 27 Aug 2014, final revision 14 Jan 2015, accepted 14 Feb 2015
}

\section{Funding}

The data for this manuscript were generated from the Systematic Treatment Optimization Program for Early Mania (STOP-EM), which was supported by unrestricted grant funding from AstraZeneca Canada. The sponsor had no input into the design or conduct of the study; collection, management, analysis, or interpretation of the data; preparation, review, or approval of the manuscript; or decision to submit the manuscript for publication.

\section{References}

1 Ng M, Fleming T, Robinson M, Thomson B, Graetz N, Margono C, et al. Global, regional, and national prevalence of overweight and obesity in children and adults during 1980-2013: a systematic analysis for the Global Burden of Disease Study 2013. Lancet 2014; 384: 766-81.

2 Fagiolini A, Frank E, Scott JA, Turkin S, Kupfer DJ. Metabolic syndrome in bipolar disorder: findings from the Bipolar Disorder Center for Pennsylvanians. Bipolar Disord 2005; 7: 424-30.

3 Shah A, Shen N, El-Mallakh RS. Weight gain occurs after onset of bipolar illness in overweight bipolar patients. Ann Clin Psychiatry 2006; 18: 239-41.

4 Fiedorowicz JG, Palagummi NM, Forman-Hoffman VL, Miller del D, Haynes WG. Elevated prevalence of obesity, metabolic syndrome, and cardiovascular risk factors in bipolar disorder. Ann Clin Psychiatry 2008; 20: 131-7.

5 Sicras A, Rejas J, Navarro R, Serrat J, Blanca M. Metabolic syndrome in bipolar disorder: a cross-sectional assessment of a Health Management Organization database. Bipolar Disord 2008; 10: 607-16.

$6 \mathrm{Kim} \mathrm{B}$, Kim SJ, Son Jl, Joo YH. Weight change in the acute treatment of bipolar I disorder: a naturalistic observational study of psychiatric inpatients. J Affect Disord 2008; 105: 45-52.

7 Lee NY, Kim SH, Cho B, Lee YJ, Chang JS, Kang UG, et al. Patients taking medications for bipolar disorder are more prone to metabolic syndrome than Korea's general population. Prog Neuropsychopharmacol Biol Psychiatry 2010; 34: 1243-9.

8 Goldstein BI, Liu SM, Zivkovic N, Schaffer A, Chien LC, Blanco C. The burden of obesity among adults with bipolar disorder in the United States. Bipolar Disord 2011; 13: 387-95.

9 Ouchi N, Parker JL, Lugus JJ, Walsh K. Adipokines in inflammation and metabolic disease. Nat Rev Immunol 2011; 11: 85-97.

10 Uysal KT, Wiesbrock SM, Marino MW, Hotamisligil GS. Protection from obesity-induced insulin resistance in mice lacking TNF-alpha function. Nature 1997; 389: 610-4.

11 Brain Development Cooperative Group. Total and regional brain volumes in a population-based normative sample from 4 to 18 years: the NIH MRI Study of Normal Brain Development. Cereb Cortex 2012; 22: 1-12.

12 Gunstad J, Paul RH, Cohen RA, Tate DF, Spitznagel MB, Gordon E. Elevated body mass index is associated with executive dysfunction in otherwise healthy adults. Compr Psychiatry 2007; 48: 57-61.

13 Anstey KJ, Cherbuin N, Budge M, Young J. Body mass index in midlife and late-life as a risk factor for dementia: a meta-analysis of prospective studies. Obes Rev 2011; 12: e426-37.

14 Colman RJ, Anderson RM, Johnson SC, Kastman EK, Kosmatka KJ, Beasley TM, et al. Caloric restriction delays disease onset and mortality in rhesus monkeys. Science 2009; 325: 201-4.

15 Siervo M, Arnold R, Wells JC, Tagliabue A, Colantuoni A, Albanese E, et al. Intentional weight loss in overweight and obese individuals and cognitive function: a systematic review and meta-analysis. Obes Rev 2011; 12: 968-83.

16 Fagiolini A, Kupfer DJ, Houck PR, Novick DM, Frank E. Obesity as a correlate of outcome in patients with bipolar I disorder. Am J Psychiatry 2003; 160: $112-7$.

17 Kemp DE, Gao K, Chan PK, Ganocy SJ, Findling RL, Calabrese JR. Medical comorbidity in bipolar disorder: relationship between illnesses of the endocrine/metabolic system and treatment outcome. Bipolar Disord 2010; 12: 404-13.

18 Depp CA, Strassnig M, Mausbach BT, Bowie CR, Wolyniec $\mathrm{P}$, Thornquist MH, et al. Association of obesity and treated hypertension and diabetes with cognitive ability in bipolar disorder and schizophrenia. Bipolar Disord 2014; 16: 422-31.

19 Bond DJ, Ha TH, Lang DJ, Su W, Torres IJ, Honer WG, et al. Body mass indexrelated regional gray and white matter volume reductions in first-episode mania patients. Biol Psychiatry 2014; 76: 138-45.

20 Kuswanto CN, Sum MY, Yang GL, Nowinski WL, McIntyre RS, Sim K. Increased body mass index makes an impact on brain white-matter integrity in adults with remitted first-episode mania. Psychol Med 2014; 44: 533-41.

21 Gigante AD, Bond DJ, Lafer B, Lam RW, Young LT, Yatham LN. Brain glutamate levels measured by magnetic resonance spectroscopy in patients with bipolar disorder: a meta-analysis. Bipolar Disord 2012; 14: 478-87. 
22 Yatham LN, Kauer-Sant'anna M, Bond DJ, Lam RW, Tam E. Course and outcome after the first manic episode in patients with bipolar disorder: prospective 12-month data from the Systematic Treatment Optimization Program for Early Mania (STOP-EM) project. Can J Psychiatry 2009; 54: 105-12.

23 American Psychiatric Association. Diagnostic and Statistical Manual of Mental Disorders (4th edn, text revision) (DSM-IV-TR). APA, 2000.

24 Yatham LN, Kennedy SH, Parikh SV, Schaffer A, Beaulieu S, Alda M, et al. Canadian Network for Mood and Anxiety Treatments (CANMAT) and International Society for Bipolar Disorders (ISBD) collaborative update of CANMAT guidelines for the management of patients with bipolar disorder: update 2013. Bipolar Disord 2013; 15: 1-44.

25 Sheehan DV, Lecrubier $\mathrm{Y}$, Sheehan $\mathrm{KH}$, Amorim $\mathrm{P}$, Janavs J, Weiller $\mathrm{E}$, et al The Mini-International Neuropsychiatric Interview (M.I.N.I.): the development and validation of a structured diagnostic psychiatric interview for DSM-IV and ICD-10. J Clin Psychiatry 1998; 59 (suppl 20): 22-33.

26 Young RC, Biggs JT, Ziegler VE, MeyerDA. A rating scale for mania: reliability, validity and sensitivity. Br J Psychiatry 1978; 133: 429-35.

27 Montgomery SA, Åsberg M. A new depression scale designed to be sensitive to change. Br J Psychiatry 1979; 134: 382-9.

28 Overall JE, Gorham DR. The Brief Psychiatric Rating Scale. Psychol Reports 1962; 10: 799-812.

29 Smith SM, Jenkinson M, Woolrich MW, Beckmann CF, Behrens TE, Johansen-Berg $\mathrm{H}$, et al. Advances in functional and structural MR image analysis and implementation as FSL. Neuroimage 2004; 23 (suppl 1): S208-19.

30 Woolrich MW, Jbabdi S, Patenaude B, Chappell M, Makni S, Behrens T, et al Bayesian analysis of neuroimaging data in FSL. Neuroimage 2009; 45 (suppl 1): S173-86.

31 Posse S, Otazo R, Caprihan A, Bustillo J, Chen H, Henry PG, et al. Proton echo-planar spectroscopic imaging of J-coupled resonances in human brain at 3 and 4 Tesla. Magn Reson Med 2007; 58: 236-44.

32 Guh DP, Zhang W, Bansback N, Amarsi Z, Birmingham CL, Anis AH. The incidence of co-morbidities related to obesity and overweight: a systematic review and meta-analysis. BMC Public Health 2009; 9: 88.

33 Prospective Studies Collaboration. Body-mass index and cause-specific mortality in 900000 adults: collaborative analyses of 57 prospective studies. Lancet 2009; 373: 1083-96.
34 Berrington de Gonzalez A, Hartge P, Cerhan JR, Flint AJ, Hannan L, Macinnis RJ, et al. Body-mass index and mortality among 1.46 million white adults. N Engl J Med 2010; 363: 2211-9.

35 Wurtz $\mathrm{P}$, Wang Q, Kangas AJ, Richmond RC, Skarp J, Tiainen $\mathrm{M}$, et al. Metabolic signatures of adiposity in young adults: mendelian randomization analysis and effects of weight change. PLOS Med 2014; 11: e1001765.

36 Goldstein BI, Kemp DE, Soczynska JK, McIntyre RS. Inflammation and the phenomenology, pathophysiology, comorbidity, and treatment of bipolar disorder: a systematic review of the literature. J Clin Psychiatry 2009; 70: 1078-90.

37 Capuron L, Miller AH. Immune system to brain signaling: neuropsychopharmacological implications. Pharmacol Ther 2011; 130: 226-38.

38 Rao JS, Harry GJ, Rapoport SI, Kim HW. Increased excitotoxicity and neuroinflammatory markers in postmortem frontal cortex from bipolar disorder patients. Mol Psychiatry 2010; 15: 384-92.

39 Haarman BC, Riemersma-Van der Lek RF, de Groot JC, Ruhe HG, Klein HC, Zandstra TE, et al. Neuroinflammation in bipolar disorder - A [C]-(R)-PK11195 positron emission tomography study. Brain Behav Immun 2014; 40: 219-25.

40 Kurt E, Guler O, Serteser M, Cansel N, Ozbulut O, Altinbaș K, et al. The effects of electroconvulsive therapy on ghrelin, leptin and cholesterol levels in patients with mood disorders. Neurosci Lett 2007; 426: 49-53.

41 Harvey J. Leptin: a diverse regulator of neuronal function. J Neurochem 2007; 100: 307-13.

42 Andrews ZB. The extra-hypothalamic actions of ghrelin on neuronal function. Trends Neurosci 2011; 34: 31-40.

43 Signore AP, Zhang F, Weng Z, Gao Y, Chen J. Leptin neuroprotection in the CNS: mechanisms and therapeutic potentials. J Neurochem 2008; 106 1977-90.

44 Harvey J, Solovyova N, Irving A. Leptin and its role in hippocampal synaptic plasticity. Prog Lipid Res 2006; 45: 369-78.

45 Opland DM, Leinninger GM, Myers Jr MG. Modulation of the mesolimbic dopamine system by leptin. Brain Res 2010; 1350: 65-70.

46 Porter RK AJ. Effects of leptin on mitochondrial 'proton leak' and uncoupling proteins: implications for mammalian energy metabolism. Proc Nutr Soc 1998; 57: 455-60. 